\title{
Permeability measurements using oscillatory flows
}

\author{
Baudouin Géraud • Jerome A. Neufeld • \\ Paul R. Holland · M. Grae Worster
}

Received: date / Accepted: date

\begin{abstract}
We describe a versatile apparatus for measuring the permeability of porous materials using oscillatory flows. The permeabilities are measured by an original spectral analysis of the pressure and fluid-displacement signals. The measurements are shown to be in very good agreement with classical drainage experiments, performed on the same device. Our apparatus and methodology will be useful if small fluid displacements are required, for example in reactive porous media.

Include keywords, PACS and mathematical subject classification numbers as
\end{abstract} needed.

Keywords Permeability · Oscillating flows · Spectral analysis

\section{Contents}

1 Introduction . . . . . . . . . . . ............. 2

2 Experimental apparatus and protocol ...................... 3

3 Modelling and signal processing . . . . . . . . . . . . . . . . . . . . . . 9

4 Results and observations . . . . . . . . . . . . . . . . . . . . . . . . . . . 14

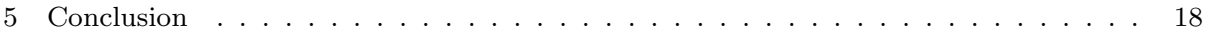

A Drainage experiments . . . . . . . . . . . . . . . . . . . . . . . . 20

B Analysis of the signal Harmonics . . . . . . . . . . . . . . . . . . . . . . . . . . . . . 20

B. Géraud · M.G. Worster

Department of Applied Mathematics and Theoretical Physics, Wilberforce Road, Cambridge CB3 0WA, UK

E-mail: mgw1@cam.ac.uk E-mail: bgeraud.pro@gmail.com

J.A. Neufeld

BP Institute, Bullard Laboratories, Madingley Road, Cambridge CB3 0EZ, UK

Department of Applied Mathematics and Theoretical Physics, Wilberforce Road, Cambridge CB3 0WA, UK

Department of Earth Sciences, University of Cambridge

Tel.: +44-1-223-765709

E-mail: j.neufeld@bpi.cam.ac.uk

P.R. Holland

British Antarctic Survey, High Cross, Madingley Rd, Cambridge CB3 0ET, UK

E-mail: pahol@bas.ac.uk 


\section{Introduction}

Porous media are ubiquitous in the natural environment and industrial applications. A fundamental characteristic of porous media is their permeability $K$, which relates the volume flux per unit area $\mathbf{v}$ through a medium to the pressure gradient applied across it and the body force, such as gravity, acting on the fluid within it, according to Darcy's law

$$
\mathbf{v}=-\frac{K}{\mu}(\nabla P-\rho \mathbf{g}),
$$

where $\mu$ is the dynamic viscosity of the pore fluid, $\rho$ is its density, $P$ is the local pore pressure and $\mathbf{g}$ is the acceleration due to gravity. Therefore the inverse of the permeability coefficient characterises the resistance to flow through a prorous medium under a fixed pressure gradient. Although there are some semi-empirical relationships, such as the Carmen-Kozeny equation [1], used to estimate the permeability of a porous medium from knowledge of its porosity, the permeability depends significantly on the internal morphology of the medium and must be measured in most practical applications.

Permeability is usually measured by applying a known pressure difference $\Delta P$ across a sample of length $L$ and cross-section $S$ and measuring the volume flux $Q$ of a pore fluid of known viscosity $[2,3]$. The permeability is then given by the formula

$$
K=\frac{\mu Q / S}{\Delta P / L}
$$

This method is simple and robust but relies on a sustained flow through the porous medium being measured. However, if the porous medium is reactive then the pore size and hence its permeability can evolve while a measurement is being made in consequence of the sustained flow carrying heat and chemicals through the medium. An example is a mushy layer such as sea ice, which is a reactive porous medium made up of dendritic crystals formed during the freezing of alloys. The crystals can redissolve in response to flow of the pore liquid in the direction of any applied temperature field. The disturbance of the gradients in brine salinity by a uni-directional flow can also lead to variations of the permeability of several orders of magnitude [4-7]. Additionally in some systems containing fine particles, a sustained flow can lead to pore clogging and result in an important decrease of the permeability during the measurement [3]. These observations indicate that the classical method to measure permeabilities with a sustained flow may not be sufficient for systems whose properties depend strongly on local gradients or on the mobilisation of the particles they contain.

Here, we describe our development of a new experimental apparatus to measure permeability that uses an oscillating flow of small displacement and thus avoids the imposition of a sustained uni-directional flow. We reverse the usual procedure by controlling the flow and measuring the consequent pressure drop across the sample. Spectral analysis of the measured signals allows for precise control of measurement errors and has the added potential (by recording phase differences) to measure characteristics of deformable poro-elastic media.

This paper is focused on detailing the experimental setup and the spectral analysis used to determine permeabilities. As a preliminary test of the technique, we report on experiments using packed beads of glass. We validate our results by making supplementary measurements using the classical drainage technique. 


\section{Experimental apparatus and protocol}

Central to our experimental design is the aim to produce an oscillating flow through a porous medium in order to measure its permeability without the imposition of a mean flow. The flow is driven by a rigid piston of cross-sectional area $S_{p}$ whose displacement $D_{p}(t)$ is measured over time. This measurement determines the flow rate through the flow cell by volume conservation $Q(t)=S_{p} d D_{p}(t) / d t$. The pressure drop across the porous medium $\Delta P(t)$ is measured with a Differential Pressure Sensor (DPS). The pressure drop and flow rate signals are then used to compute the hydraulic resistance of the flow cell $R_{H}$ and the permeability of the porous medium.

\subsection{Experimental setup}

The experimental apparatus comprises several elements, shown in figure 1 and detailed below.

\subsubsection{Flow generation}

Oscillating flows are generated by a rigid piston below the flow cell connected to a shaker (Data Physics SignalForce V20) supplied by a power amplifier (Data Physics SignalForce PA100E) and controlled by an acquisition card (Data Physics Quattro). The piston is screwed to the moving part of the shaker through a thick $(6 \mathrm{~mm})$ steel plate. A displacement transducer is screwed to the steel plate to measure the piston stroke $D_{p}(t)$ as a function of time. Our experiments were performed at a single frequency $f_{0}=1 \mathrm{~Hz}$, chosen to be low enough to avoid vibration of the support frame and to keep the pore Reynolds number small. The pore Reynolds number $R e_{p}=\rho v_{p} d / \mu$, where $v_{p}$ is a characteristic magnitude of the pore velocity and $d$ is a characteristic size of the pores, measures the ratio of viscous stresses to inertia. It must be small in order for Darcy's equation to be valid, and this is achieved by ensuring that the pore velocity, of the order of $v_{p} \approx D_{0} f_{0} / \phi$ (where $D_{0}$ is a characteristic amplitude of $D_{p}(t)$ and $\phi$ the porosity) is sufficiently small in our expriments.

\subsubsection{Flow cell}

The flow cell is a perspex cylinder with a $65 \mathrm{~mm}$ internal diameter. The porous medium is held between two metal plates drilled with regularly spaced $0.7 \mathrm{~mm}$ diameter holes to ensure that the fluid enters the porous medium homogeneously. The meshes are covered with a silk fabric to avoid the loss of the smallest beads and are supported by two inner perspex cylinders (A and B on Fig. 1), inserted in the flow cell. Cylinder (A) keeps the porous medium away from the flow injection and ensures that the flow is unidirectional before entering the porous medium. The cylinder (B) presses the upper mesh with a $5.65 \mathrm{~kg}$ mass to compact the glass beads and avoid the fluidisation of the bed [8] when the flow velocity is directed upwards. Another, cautionary mesh is set at the bottom of the flow cell to prevent beads from dropping into the piston. 


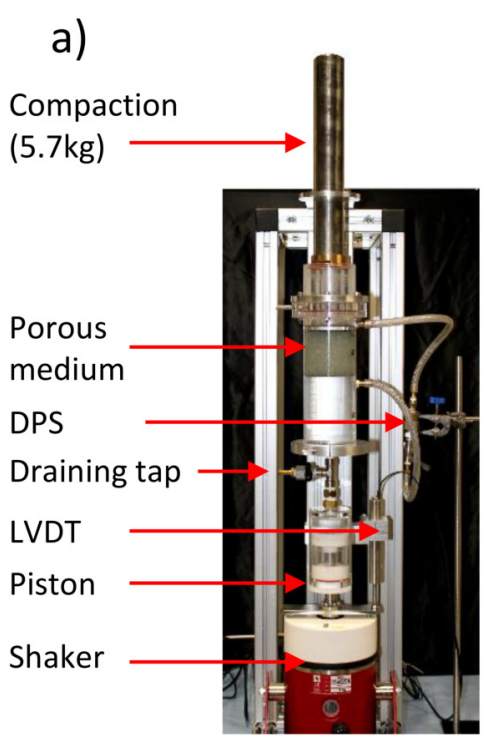

b)

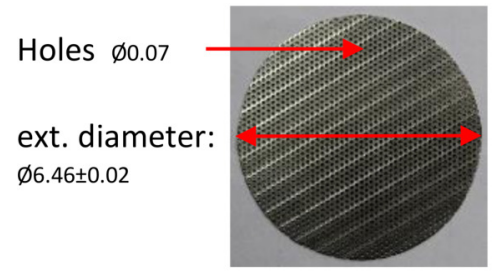

c)

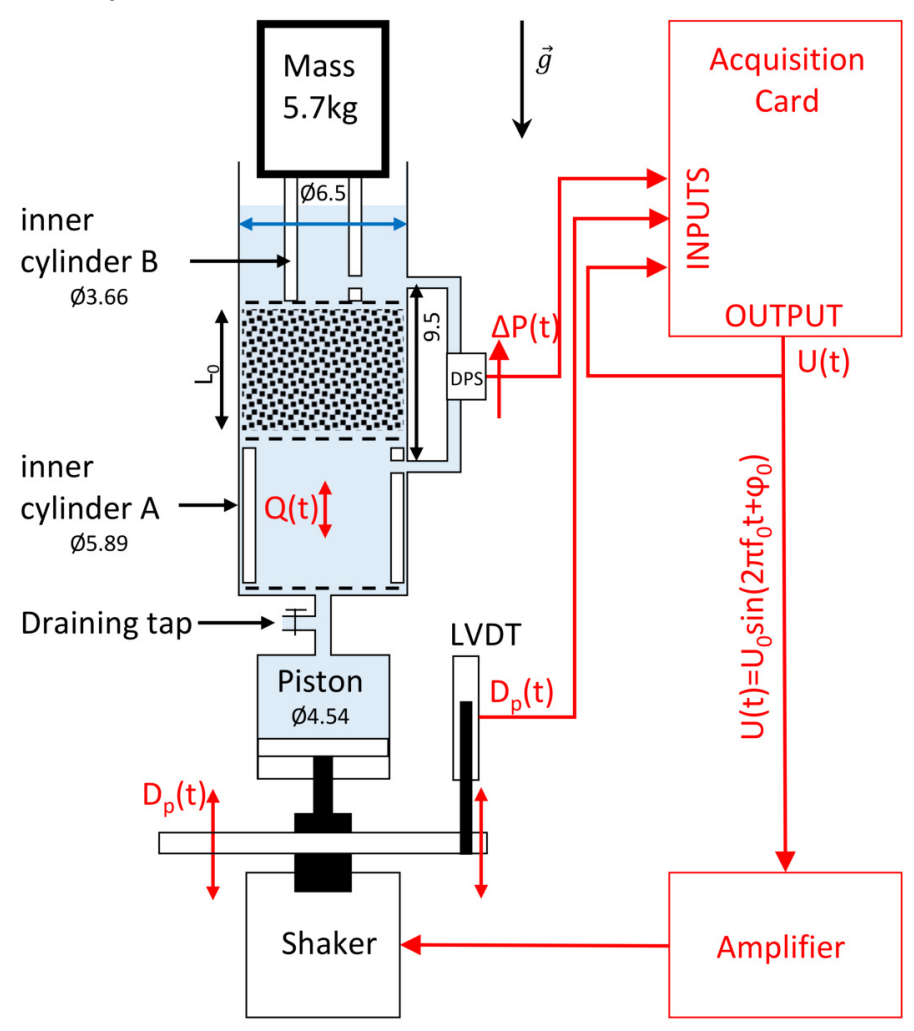

Fig. 1 Photos and sketch of the experimental setup. a): photo of the device. The metal frame in background reduces any vibration of the device during the experiments. The Linear Variable Differential Transformer (LVDT) and the Differential Pressure Sensor (DPS) are situated on the right-hand side. $\mathbf{b}$ ): photo of a mesh used to maintain the grains in the flow cell. The mean diameter is $64.6 \mathrm{~mm}$ and the typical hole radius is $0.7 \mathrm{~mm}$. Each mesh was covered with silk fabric to retain the smallest grains. c): sketch of the device in cross-sectional view. The region accessible to the fluid (water) is colored in light blue and the acquisition circuit is shown in red. All the dimensions are indicated in $\mathrm{mm}$, the diameters are indicated with double arrows and the symbols $\varnothing$. The porous medium is compacted between two meshes borne by two perspex cylinders (A and B), drilled for pressure measurements. The DPS measures the pressure drop across the porous medium of length $L_{0}$. The LVDT measures the piston stroke and therefore its displacement $D_{p}(t)$, proportional to the displacement of the fluid in the flow cell $D(t)$ by volume conservation. The flux $Q(t)$ is computed from the measurement of $D_{p}(t)$ (see section 2.3). 

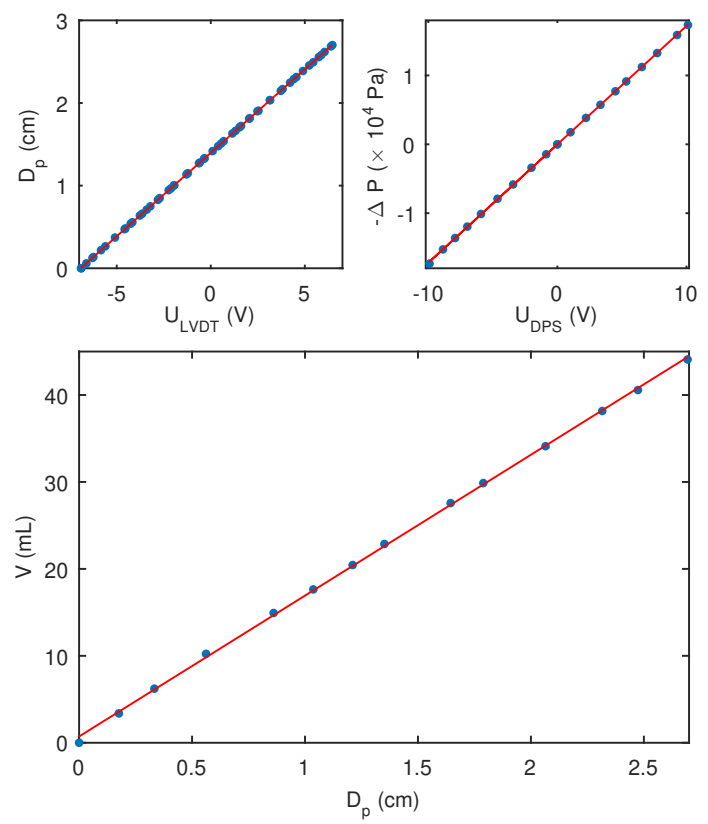

Fig. 2 Calibration curves of the sensors and the piston. The experimental points are in blue, the red lines are linear fits. a) Displacement transducer calibration. Sensitivity: $2.0192 \pm 0.0005 \mathrm{~mm} / \mathrm{V}$. b) Differential Pressure Sensor calibration. Sensitivity: $1719 \pm 3 \mathrm{~Pa} / \mathrm{V}$. c) Measurement of the volume of liquid displaced in the flow cell for several piston positions. The linear fit provides the section of the piston and so its diameter with an excellent precision: $45.4 \pm 0.3 \mathrm{~mm}$.

\subsubsection{Sensors: calibration and sensitivity}

The acquisition card records the piston stroke and the pressure drop between two points at the inlet and the outlet of the porous medium simultaneously. This pressure drop $\Delta P$ is measured with a differential pressure sensor (DPS, Omega engineering, customized online: wet/wet differential, bidirectional, range 0.17bar, output $\pm 10 \mathrm{VDC})$, connected to the flow cell through meshed, rigid hoses. It is important to note that there is no flow through the DPS so that the pressure drops between the flow cell and each inlet of the sensor are hydrostatic.

The displacement transducer is a Linear Variable Differential Transformer (LVDT, Omega engineering LD620-10), which enables accurate measurements of the displacement of the piston in time $D_{p}(t)$, proportional to the volume of fluid displaced in the flow cell $V(t)=S_{p} D_{p}(t)$, with $S_{p}$ the cross-sectional area of the piston. The linearity of both sensors was checked with calibration experiments performed by measuring constant displacements or hydrostatic pressures over the whole range of the sensors (Fig. 2). These calibration experiments determined the relative uncertainties of the measurements of the pressure and the volume of fluid displaced which are respectively $0.17 \%$ and $1.3 \%$. We could then measure $S_{p}=1.62 \times 10^{-3} \mathrm{~m}^{2}$ accurately by regression, as it is a crucial parameter for the measurements of the 


\begin{tabular}{cccc}
\hline beads & $d(\mathrm{~mm})$ & $L_{0}(\mathrm{~mm})$ & $\phi$ \\
\hline A & $0.12 \pm 0.03$ & 75.0 & 0.405 \\
B & $0.37 \pm 0.01$ & 76.0 & 0.407 \\
C & $0.47 \pm 0.01$ & 75.0 & 0.392 \\
D & $0.68 \pm 0.01$ & 76.5 & 0.423 \\
E & $1.07 \pm 0.01$ & 75.0 & 0.395 \\
F & $1.44 \pm 0.01$ & 75.0 & 0.393 \\
G & $2.20 \pm 0.03$ & 76.5 & 0.377 \\
\hline
\end{tabular}

Table 1 Summary table of the several porous media parameters used for the experiments. The porous media are composed of packed glass beads of average diameter $d$ and porosity $\phi$. The length $L_{0}$ corresponds to the height of the grain pile in the flow cell.

volume of fluid displaced $V(t)$ and therefore for the computation of the flow rate $Q(t)$.

\subsubsection{Porous media}

To calibrate, test and characterize this new technique, we used porous media made of compacted glass beads (ballotini) placed in the flow cell between the two meshes. Great care was taken to ensure that no bubbles were trapped during the setup and the filling of the device ${ }^{1}$. We varied the bead diameters to probe a range of permeabilities spanning several orders of magnitude. The density and the total volume of grains introduced in the flow cell were measured by weighing to determine the porosities of the porous media. We measured the length of the porous medium $L_{0}$ to estimate its porosity $\phi$ of the order of 0.400 with a fair precision of $\Delta \phi \approx \pm 0.015$, which corresponds to a relative uncertainty of $\pm 4 \%$. The table 1 summarizes the different experimental parameters probed in this study. Each pack of beads is denoted by a letter (A-G) to which we will refer in the following.

\subsection{Experimental protocol}

We applied a sequence of quick oscillations with the shaker at $10 \mathrm{~Hz}$ to ensure a good compaction of the packed beads before each series of experiments. The fluid used was water, with a dynamic viscosity $\mu=1.05 \mathrm{mPa}$. The shaker device is an electrodynamic one which generates forces in a moving assembly by driving an electrical coil in a magnetic field [9]. The piston motion, driven by this force generator, reacts accordingly to the piston stiffness and some experiments present slow transients in which the amplitude of the piston stroke decreases slowly. We checked for these experiments that the transient had no effect on the permeability measurements. Indeed, the permeabilities measured with full signals and with truncated ones (without the transient) only differ by $0.4 \%$, which is less that the experimental uncertainties.

1 Particularly, the beads were stirred with a rod and the absence of air was always checked by a visual inspection from the top and the sides of the device before compressing the grains. 
The oscillations of the piston and the signal acquisition are both controlled with the digital acquisition card (DAQ). The duration of each experiment was 512s and the sampling acquisition frequency was $64 \mathrm{~Hz}$. The acquisition card generates an input voltage $U(t)$ to drive the motion of the shaker. In our experiments, $U(t)$ was a sine wave of frequency $f_{0}=1 \mathrm{~Hz}$ and arbitrary phase shift $\varphi_{0}$, of which we varied the amplitude $U_{0}$.

The experiments were performed by varying the amplitude $U_{0}$ to the amplifier to probe several flow velocities with the same porous medium. Drainage experiments in which the fluid was allowed to drain through the porous medium under a prescribed hydrostatic head were performed just after each oscillating run. We could then compare the values of permeability measured with oscillations to a classical technique (see appendix A).

\subsection{Signal acquisition}

A typical example of the signals acquired is shown in figure 3, which displays the shaker's input voltage $U(t)$ (Fig. 3a) with both measurements of the pressure drop $\Delta P(t)$ (Fig. 3b) and the volume of fluid displaced $V(t)$ (Fig. 3c). Although the input voltage of the shaker is a sine wave, its output motion is not sinusoidal but saturates between two positions. This is due to the fact that the shaker is a force generator, reacting to the stiffness of the piston and the flow. As a consequence the extremal values of $U(t)$, marked in vertical dashed lines in Fig. 3 correspond to the extremal values of $\Delta P(t)$. However, this behaviour does not impact the permeability measurement since both signals $\Delta P(t)$ and $V(t)$ are recorded at the same time. The shape of these signals is such that the time scales of the rise and the fall of the piston are much smaller than the period. This allows the generation of flows with high velocities and small displacements of the fluid. The amplitude of the displacement of the fluid inside the porous medium is indeed below $1 \mathrm{~mm}$ in the example of figure 3c). This point may be of interest for some applications of this technique to permeability measurements for which the solid phase is in thermal or chemical equilibrium with the liquid.

\subsection{Flow regime and Reynolds number}

As the piston displaces the fluid (water) along the flow cell, the filtration velocity $v(t)$ varies in time and is defined as:

$$
v(t)=\frac{Q(t)}{S_{0}},
$$

where $S_{0}$ is the cross sectional area of the flow cell and $Q(t)$ the volumetric flow rate. The flow regime is characterised by the Reynolds number, defined as

$$
R e=\frac{\rho d\langle v\rangle}{\mu}
$$

where $\langle v\rangle$ is the order of magnitude of the flow velocity during an experiment. As the flow velocity $v(t)$ varies during each period of oscillation, the amplitude of the velocity $\langle v\rangle$ was defined as the average of the maximum values of velocity 
(a)

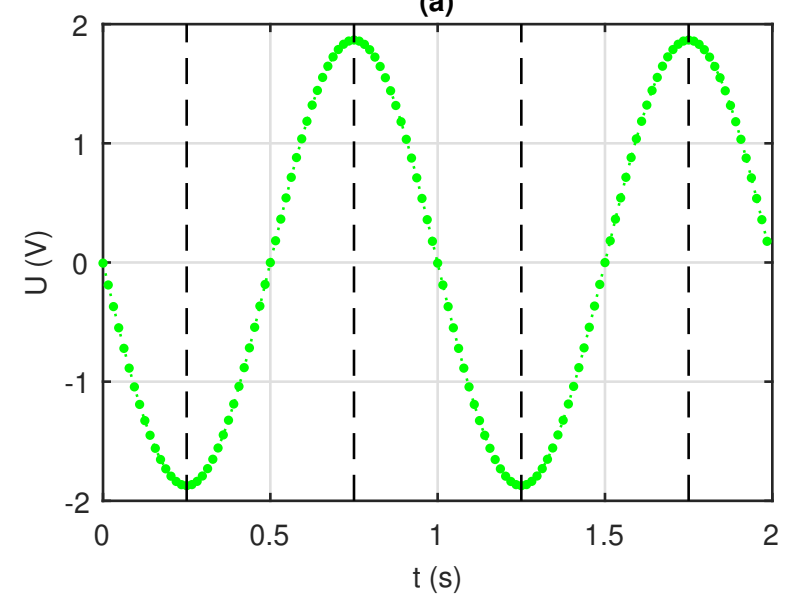

(b)

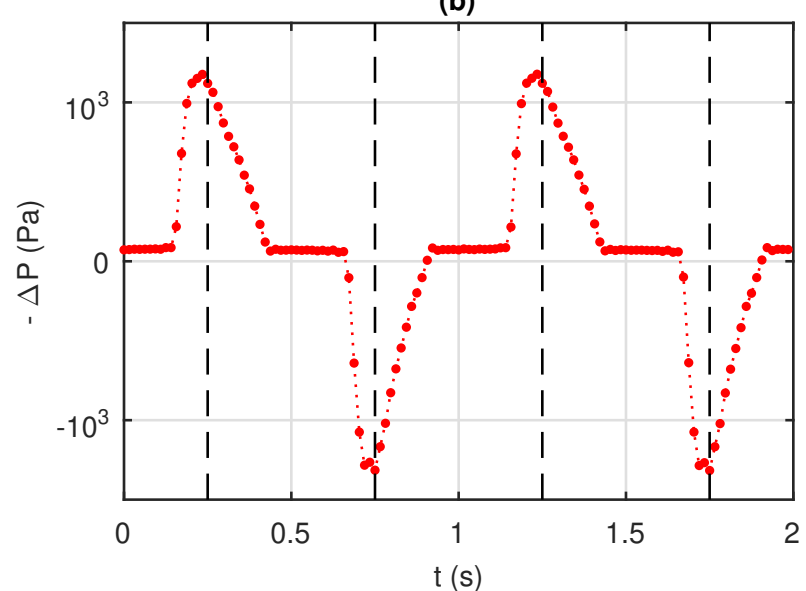

(c)

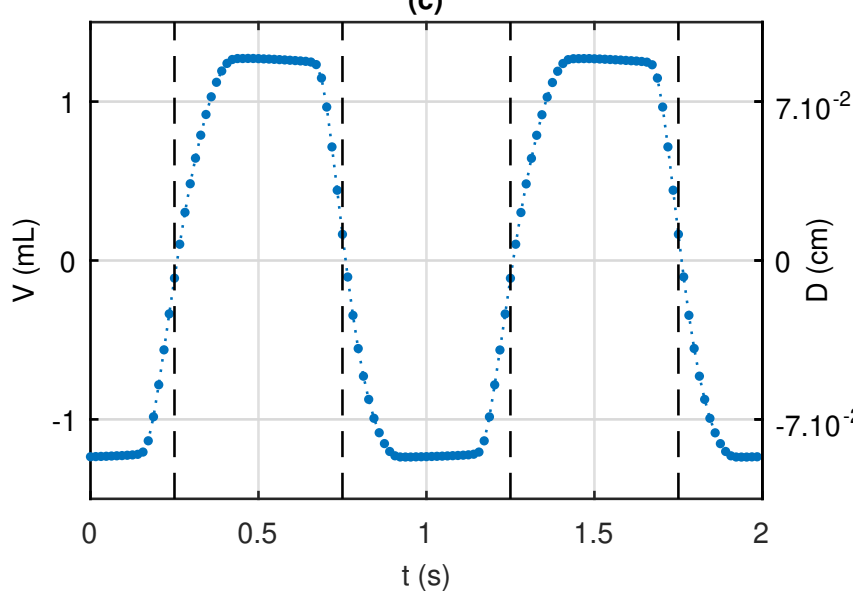

(d)

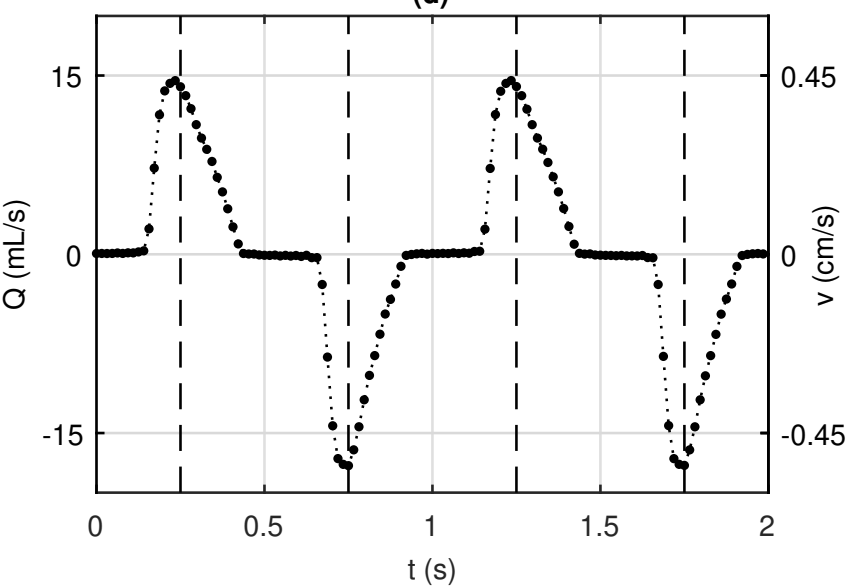

Fig. 3 Signals involved in the acquisition and the data processing. a) input voltage delivered to the shaker. The vertical dashed lines denote the positions of its extremal values on all the other graphs. The extremal values of the input voltage correspond to the extremal values of the pressure drop and the volumetric flow rate. b): Raw measurements of the pressure drop $\Delta P$, oriented opposite to gravity. c) Volume of fluid displaced in the flow cell by the piston $V(t)$ (bottom) and the corresponding displacement of the fluid inside the flow cell. d) Signal of the flux through the flow cell computed with equation 13, and the corresponding values of the filtration velocities.

amplitudes of the flow when the piston moves upwards and downwards. Alternative definitions of $\langle v\rangle$ could have been the standard deviation of $v(t)$ during a period instead or its maximal absolute value, but they proved to have very limited impact on the results described here. In our experiments using water, beads of up to $2 \mathrm{~mm}$ in diameter and fluid displacements at the pore scale between $0.1 \mathrm{~mm}$ to $2.8 \mathrm{~mm}$, the Reynolds number ranges from about 0.07 to 20 . We nevertheless find agreement with Darcy's law for Reynolds numbers up to about 10. This is consistent with well 
established results for flows in porous media [1] and indicates that the definition of $\langle v\rangle$ used for the calculation of the Reynolds number is relevant.

2.5 Quasi-static approximation and oscillation frequency

An additional requirement to the experimental protocol is to ensure that transient effects induced by the motion of the piston are negligible so that equation 1 can apply. In other words, the oscillation frequency shall be low enough for equation 1 to hold at any time, so the filtration velocity and the pressure gradient vary linearly with each other. Unsteady flows through porous media in laminar regime have been described successfully by introducing an additional term and a time delay $\tau$ in equation 1 as:

$$
\tau \partial_{t} \mathbf{v}+\mathbf{v}=-\frac{K}{\mu}(\nabla P-\rho \mathbf{g})
$$

Various models have been proposed to describe the characteristic time delay [15-17], in some of which it is even not necessarily constant[18]. However, its order of magnitude is greater and of the order of $K /(\phi \nu)$ in all the models proposed[18, 19], where $\nu=\mu / \rho$ is the kinematic viscosity of the fluid and from which we can define a characteristic frequency $f_{\tau}=\phi \nu / K$. This implies that the oscillation frequency $f_{0}$ shall be negligible compared to $f_{\tau}$ to neglect the impact of transient effects:

$$
f_{0}<<f_{\tau}=\frac{\phi \nu}{K}
$$

In our experiments, $f_{\tau}$ varies from $28 \mathrm{kHz}$ (with the smallest beads) to $121 \mathrm{~Hz}$ (with the largest ones), while $f_{0}$ had a set value of $1 \mathrm{~Hz}$. The results obtained with the largest beads show that a ratio of frequencies of about 120 proved sufficient for the quasi-static approximation to be valid, although some subtle transient effects started to be visible through a small phase shift between the pressure and velocity signals (section 4.2). These observations indicate that the oscillation frequency $f_{0}$ shall not exceed the order of $f_{\tau} / 100$ for the quasi-static approximation to be valid.

\section{Modelling and signal processing}

In this section, we detail the experimental principle and how we compute the permeability $K_{0}$ of a porous medium placed in the flow cell. We introduce the equivalent pressure drop $\Pi(t)$ and the hydraulic resistance $R_{H}$ computed from the measurements of $\Delta P(t)$ and $Q(t)$.

\subsection{Hydraulic resistance and permeability measurements}

To model the permeability measurement accurately, the flow cell can be subdivided into five regions between the positions of the two tubes connecting the differential pressure sensor. These regions are respectively the porous medium studied in the experiment, the two meshes enclosing it and the regions situated below and above 


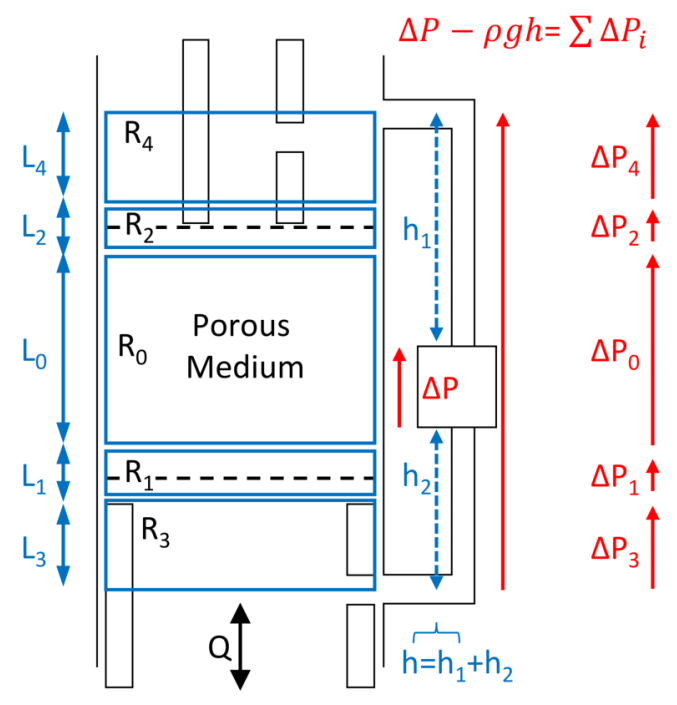

Fig. 4 Representation of the flow cell from a side view. The blue rectangles represent the hydraulic resistances corresponding to each component of the flow cell. These components are identified by their index and are characterised by their length along the flow direction $L_{i}$ and their hydraulic resistance $R_{i}$. They are respectively the porous medium (of hydraulic resistance $\left.R_{0}\right)$, the two grid meshes enclosing it $\left(R_{1}\right.$ and $\left.R_{2}\right)$ and the two flow portions linked to the pressure sensor inputs $\left(R_{3}\right.$ and $\left.R_{4}\right)$. The fluid in the pipes connected to the pressure sensor is static. The corresponding hydrostatic pressure drop is $-\rho g h$ where $h$ is the equivalent liquid height. The red arrows represent the pressure drops $\Delta P_{i}$ across each component. $\Delta P$ is the pressure difference measured by the pressure sensor and $Q$ denotes the flux across the flow cell.

them. Each region is characterised by its flow section $S_{i}$, its permeability $K_{i}$, length $L_{i}$ and its hydraulic resistance $R_{i}$ (see Fig. 4). In the following, the subscript $i=0$ refers to the porous medium inserted in the device, the indices $i=1,2$ to the grid meshes and the values $i=3,4$ to the regions below and above them. The pressure drop across the flow cell is then the sum of the several pressure drops $\Delta P_{i}$ through each part.

The displacement transducer measures the volume of fluid displaced in the flow cell $V(t)$. This allows the computation of the flow rate $Q(t)$ by Fast Fourier Transform (FFT, see eq. 13). The flow is purely vertical and so, providing the Reynolds number remains low, we can apply Darcy's law (eq. 1) along each part of the cell in the vertical direction to give

$$
Q=-\frac{1}{R_{i}}\left(\Delta P_{i}+\rho g L_{i}\right), \quad \text { where } \quad R_{i}=\frac{\mu L_{i}}{K_{i} S_{i}} .
$$

The liquid is static in the two pipes connected to the DPS and we define $h$ as the equivalent height of liquid in the hoses. The total pressure drop is therefore

$$
\sum \Delta P_{i}=-\Delta P-\rho g h
$$


and equations 7 and 8 can be combined to give

$$
\begin{aligned}
& \Pi(t)=-\Delta P(t)-\rho g\left(\sum L_{i}-h\right) \\
& \Pi(t)=R_{H} Q(t), \quad \text { where } \quad R_{H}=\sum R_{i} .
\end{aligned}
$$

Here $\Pi$ is the pressure drop across the whole cell, proportional to the flux $Q$. The constant of proportionality is the total hydraulic resistance $R_{H}$, equal to the sum of the individual resistances. Note that $\Pi$ only differs from $-\Delta P$ by a constant, which corresponds to the hydrostatic pressure drop when there is no flow. This corresponds to the non-zero value of the pressure signal identified in figure 3 when the piston reaches its extremal positions. This value is also the time average of the pressure signal: since oscillating flows are performed here, the time average value of the flow rate is zero $\langle Q\rangle_{t}=0$, so using equation $9,\langle\Delta P\rangle_{t}=\rho g\left(h-\sum L_{i}\right)$ and therefore

$$
\Pi(t)=\langle\Delta P\rangle_{t}-\Delta P(t)
$$

which makes the computation of $\Pi$ straightforward. Here \langle\rangle$_{t}$ indicates an average in time over a unit cycle.

The total hydraulic resistance $R_{H}$ is measured by processing the Fourier transforms of the signals $\Pi(t)$ and $Q(t)$ as shown in the following section. However, $R_{H}$ includes contributions that do not belong to the porous medium only but to the all the components of the flow cell. We therefore measured the hydraulic resistance of the device $R_{D}=\sum_{i>0} R_{i}$, determined by drainage experiments involving all the components of the flow cell but the porous medium. The hydraulic resistance of the device was found to be $R_{D}=1.35 \pm 0.02 \times 10^{7} \mathrm{~Pa} \mathrm{~m}^{-3} \mathrm{~s}$. This value proved to be smaller than $R_{H}$ in all our experiments, from two orders of magnitude smaller (with the smallest beads) up to the half of $R_{H}$ (with the largest ones). This contribution is eventually removed from the measurement of $R_{H}$ to find the permeability of the porous medium,

$$
K_{0}=\frac{\mu L_{0}}{S_{0}\left(R_{H}-R_{D}\right)} .
$$

An accurate measurement of the total hydraulic resistance $R_{H}$ is thus crucial to determine the permeability $K_{0}$ of the porous medium inserted in the flow cell.

\subsection{Signal Processing and spectral analysis}

This section details the processing applied to the signals to measure the permeability of the different porous media inserted in the flow cell. This processing is performed in three steps, namely the calculation of the time variations of the flow rate $Q(t)$, the calculation of the hydraulic resistance $R_{H}$ by the analysis of the Fourier spectra of $Q$ and $\Pi$, and finally the calculation of the permeability $K_{0}$ along with the errors associated with it. 


\subsubsection{Flow rate}

The flow rate through the flow cell $Q(t)$ is computed by the Fast Fourier Transform (FFT, denoted $\mathcal{F}[]$.$) of the volume displaced V(t)$ :

$$
Q(t)=\partial_{t} V(t)=\mathcal{F}^{-1}[2 i \pi f \mathcal{F}[V(t)]]
$$

where $\mathcal{F}^{-1}[$.$] is the inverse FFT and f$ the frequency. The result is shown in figure $3 \mathrm{~d}$, which also indicates the magnitude of the filtration velocity $v(t)$. The shape of the computed signal is very similar to the pressure signal $\Delta P(t)$, although the pressure has a non-zero plateau when the flux is zero.

\subsubsection{Hydraulic Resistance}

In this section, we analyse the pressure and flux signals spectrally to measure the hydraulic resistance $R_{H}$ and deduce the permeability of the porous medium $K_{0}$ from it (eq. 12). We first compute the signals FFT, functions of the frequencies $f$ and denoted $\tilde{Q}(f)$ and $\tilde{\Pi}(f)$. Ideally, both signals would be proportional to each other (eq. 10) but in practice they are affected by some measurement noise $n$. They even may have a slight phase shift $\varphi=2 \pi f_{0} t_{0}$ due to experimental artifacts (inertial effects, compliance), so that $\tilde{\Pi}(f)$ can be expressed as

$$
\tilde{\Pi}(f)=R_{H} e^{-i 2 \pi f t_{0}} \tilde{Q}(f)+\tilde{n} .
$$

Both signals are subject to noise, and the best way to compute $Z_{H}$ without bias $[10,11]$ is with the formula

$$
R_{H}=\frac{\left\langle\left|\tilde{\Pi} \cdot \tilde{Q}^{*}\right|\right\rangle_{f}}{\left\langle\tilde{Q} \cdot \tilde{Q}^{*}\right\rangle_{f}}
$$

where the brackets $\langle.\rangle_{f}$ represent averages over the sample frequencies and the stars * refer to the complex conjugates of the Fourier transforms. The correlation between the two Fourier transforms is quantified by the normalized cross-correlation coefficient

$$
\gamma^{2}=\frac{\left\langle\tilde{Q} \cdot \tilde{\Pi}^{*}\right\rangle_{f}^{2}}{\left\langle\tilde{\Pi} \cdot \tilde{\Pi}^{*}\right\rangle_{f} \cdot\left\langle\tilde{Q} \cdot \tilde{Q}^{*}\right\rangle_{f}} .
$$

A typical spectral analysis is shown in figure 5 , where we compare the spectrum of $\Pi$ with the computation of $R_{H} Q$. The data present harmonics of the oscillating frequency $\left(f_{0}=1 \mathrm{~Hz}\right)$ for which both spectra match up within about $25 \mathrm{~Hz}$. For the porous media studied here, the spectra present an excellent matching, characterized by a normalized cross-correlation coefficient of $\gamma^{2}=0.9989$. Note that no fit is involved in the processing, all the relevant quantities $R_{H}$ and the spectra $\tilde{Q}(f)$ and $\tilde{\Pi}(f)$ are computed from the experimental data.

The normalized cross correlation coefficient is also used to asses the uncertainties[10] associated with the measurement of $R_{H}$ :

$$
\frac{\Delta R_{H}}{R_{H}}=1.96 \sqrt{\frac{\left(1-\gamma^{2}\right)}{2\left(N \gamma^{2}-1\right)}},
$$

where $N$ is the number of points used for the calculation of equations 14 and 15 , typically $N=2^{15}$. The measurement uncertainties associated with the measurement of $K_{0}$ involve the relative uncertainty of the measurement of $R_{H}$ as shown in the next paragraph. 


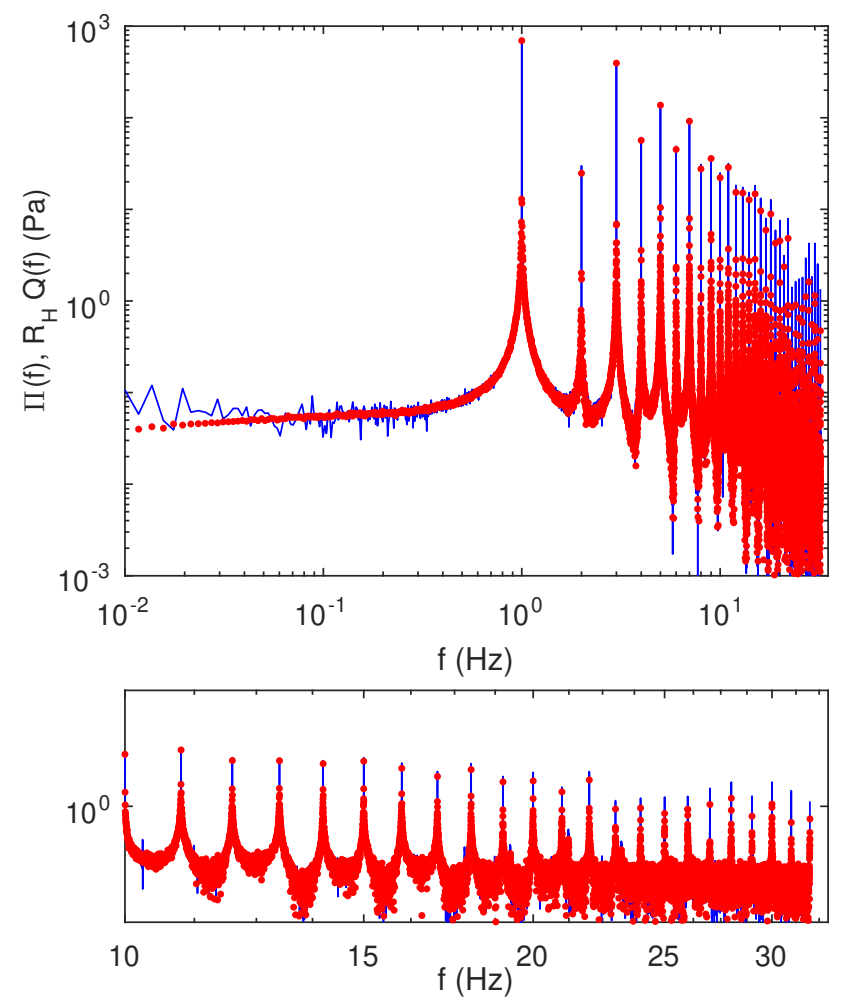

Fig. 5 Comparison of the spectra of the pressures $\Pi$ (blue line) and $R_{H} Q$ (red dots). The bottom graph is a close up on high frequencies. In this example, we have $R_{H}=7.60 .10^{7} \pm$ 2.8.10 ${ }^{4}$ Pa.s. $m^{-3}$ and the normalized cross correlation coefficient between the two signals is $\gamma^{2}=0.9989$. The signals analysed here are the same as figure 3 .

3.3 Permeability measurement and associated uncertainties.

The permeability of the porous media is finally computed using equation 12 . This equation includes the hydraulic resistance of the device, measured to be $R_{D}=$ $1.35 \pm 0.03 \times 10^{7} \mathrm{~Pa} . \mathrm{m}^{-3} \mathrm{~s}$ with an empty device by successive drainage experiments as a calibration. The measurement uncertainty associated to equation 12 is then estimated by:

$$
\frac{\Delta K_{0}}{K_{0}}=\sqrt{\left(\frac{\Delta L_{0}}{L_{0}}\right)^{2}+\left(\frac{\Delta S_{0}}{S_{0}}\right)^{2}+\left(\frac{\Delta R_{D}}{R_{D}}\right)^{2}+\left(\frac{\Delta R_{H}}{R_{H}}\right)^{2}} .
$$

Equation 17 assumes that the uncertainties are independent and neglects the uncertainties associated with $\mu$ (as experiments were performed at a constant temperature set by a thermostat). The term relative to the uncertainty in $R_{H}$ is estimated by using equation 16 , and is relatively small as $\gamma \approx 1$ and $N$ is large. Over all our experiments, we found the uncertainty in $R_{H}$ to be $0.1 \%$ in average, 
with a maximum value of $0.7 \%$ for one experiment performed with the beads $\mathrm{B}$. The dominant source of error as defined in equation 17 are then the terms related to $R_{D}$ and $L_{0}$. As mentioned at the end of paragraph 3.1, $R_{D}$ was measured with repeated experiments for a better precision. The relative uncertainty associated to this measurement is $2 \%$. The absolute uncertainty $\Delta L_{0}$ varies from $1 \mathrm{~mm}$ (smallest graduation of the height gauge) to $2 \mathrm{~mm}$ which is the maximum grain diameter, we have therefore kept this latter value to calculate the order of magnitude of the measurement uncertainty of $K_{0}$. The uncertainty on $S_{0}$ is calculated from the tolerance of the flow cell diameter which is $50 \mu \mathrm{m}$ compared to its value of $6.5 \mathrm{~mm}$. Finally, the relative uncertainty associated with the measurements of the permeability $K_{0}$ was found to be at most about $3.7 \%$. This could be improved further with more accurate measurements of $R_{D}$ and $L_{0}$.

\section{Results and observations}

In this section we present the results of the experiments and the data analysis described in sections 2 and 3. The measurements of the permeabilities of the porous media are first compared with classical measurements obtained by draining the fluid through the flow cell. The impact of the flow regimes, characterised by the Reynolds number on the measurements is then assessed and provides further insights into the experimental method. Finally, we investigate the information collated from the spectral analysis, described in section 3.

\subsection{Validation by comparison with drainage experiments}

Several bead diameters were used to span a range of permeabilities (section 2). The experiments were analysed with the processing detailed in section 3.1. For each set of packed beads (A-G), we performed the measurements with a series of flows of different amplitudes, by changing the amplitude of the shaker's input voltage $U_{0}$. The results are gathered in table 4.1. We performed drainage experiments after each oscillating experiment to compare the measurements with a reference technique. Drainage experiments have the advantage that the flows are directed downwards by gravity. They naturally and uniformaly compact the medium and do not exhibit any effect of decompaction. They are therefore a reference for the oscillatory permeability measurements. The protocol and the analysis of these experiments are described in appendix A, and the result of their comparison with oscillating measurements is shown in figure 6 .

The comparison in figure 6 shows an excellent agreement between the two measurements over a range of two orders of magnitude of permeabilities. The relative discrepancy between the two permeability measurements for a given porous medium is of the order of $3 \%$ and is essentially due to the experimental uncertainty of the method and of the estimation by drainage as well. These observations validate our experimental method as they indicate that the measurements by oscillations provide the same values of permeability as the classical technique of drainage. However, the precision of the measurements under oscillations, and the precision with which the signals have been recorded allow for a more precise characterisation of the flows. 


\begin{tabular}{|c|c|c|c|c|c|}
\hline beads & $d(m m)$ & $\left\langle K_{0}\right\rangle\left(m^{2}\right)$ & $\left\langle R_{H}\right\rangle\left(\right.$ Pa.s. $\left.m^{-3}\right)$ & $\langle\varphi\rangle$ & $\left\langle\gamma^{2}\right\rangle$ \\
\hline $\mathrm{A}$ & 0.12 & $1.4310^{-11}$ & $1.6810^{9}$ & $-2.810^{-2}$ & 0.97 \\
\hline $\mathrm{B}$ & 0.37 & $1.1210^{-10}$ & $2.3010^{8}$ & $-1.110^{-2}$ & 0.98 \\
\hline $\mathrm{C}$ & 0.47 & $1.9910^{-10}$ & $1.3310^{8}$ & $-5.210^{-3}$ & 0.97 \\
\hline $\mathrm{D}$ & 0.68 & $3.9110^{-10}$ & $7.5510^{7}$ & $2.610^{-4}$ & 0.99 \\
\hline $\mathrm{E}$ & 1.07 & $7.9610^{-10}$ & $4.3310^{7}$ & $2.910^{-3}$ & 0.99 \\
\hline $\mathrm{F}$ & 1.44 & $1.4010^{-9}$ & $3.0510^{7}$ & $8.810^{-3}$ & 0.96 \\
\hline G & 2.20 & $3.1210^{-9}$ & $2.1310^{7}$ & $2.510^{-2}$ & 0.95 \\
\hline
\end{tabular}

Table 2 Summary table of the averaged permeability measurements for each set of packed beads. The angled brackets represent the average among experiments performed with the same porous medium. The results present the measurements of the permeability $K_{0}$, the hydraulic resistance $R_{H}$, the phase shift $\varphi$ and the cross-correlation coefficient $\gamma^{2}$ between the pressure and flow rate signals.

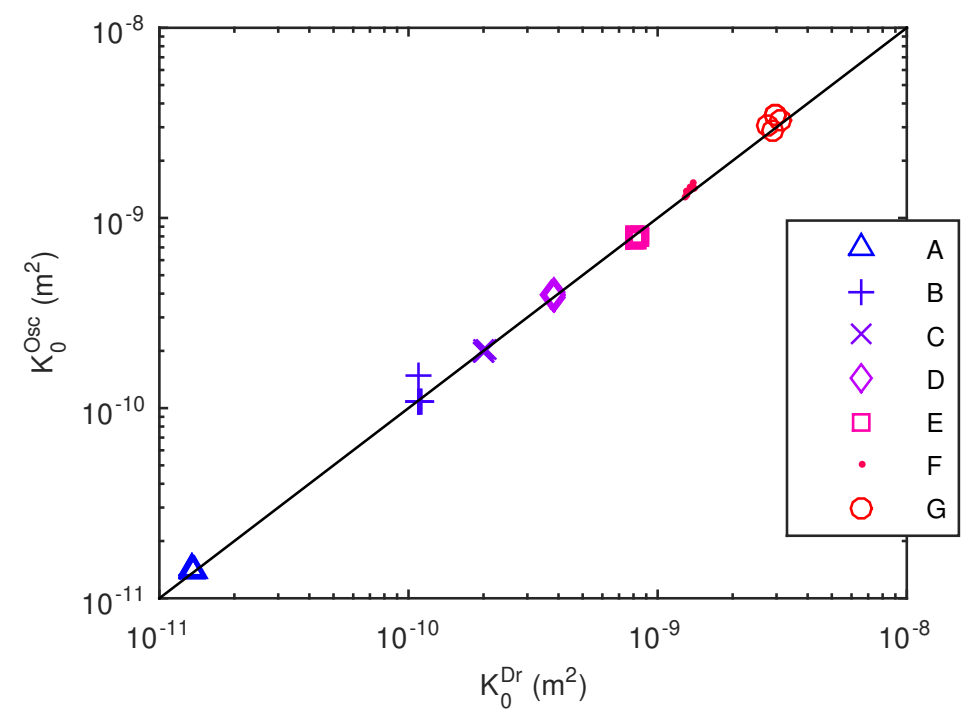

Fig. 6 Comparison between the permeabilities measured in oscillating measurements $K_{0}$ and by drainage $K^{D r}$ for 50 experiments. The black line corresponds to the straight line $K_{0}=K^{D r}$. The average discrepancy between the two measurements is $3 \%$.

\subsection{Flux variation and flow regimes}

The comparison with the drainage experiments indicates that the setup allows for accurate measurements of permeabilities using oscillating flows. However, the results do not indicate if the flow regime has an impact on the measurements or whether the assumptions made to derive equations 12 and 10 are satisfied. In particular, one can wonder if Darcy's law applies in the flow regimes probed during the experiments, which is equivalent to checking the validity of equation 10, that is $\Pi(t)=R_{H} Q(t)$, as it is directly derived from Darcy's law (section 2). 
A comparison of both signals, namely the pressure drop $\Pi(t)$ and the product $R_{H} Q(t)$, allows us then to check if the assumptions behind the analysis presented here are justified. It would also allow a check on the range of validity of the results presented in the former paragraph and to assess the impact of the flow regime on these measurements.

The flux signal $Q(t)$ is not directly measured in the flow cell but is instead computed from the measurement of the fluid displaced (see section 3). As $R_{H}$ is a constant, the signals $\Pi(t)$ and $Q(t)$ should satisfy a linear relationship if Darcy's law applies in the quasi-static regime. As the filtration velocity $v(t)$ is proportional to the flow rate, the same conclusion can be drawn if $\Pi(t)$ and $v(t)$ are in phase and proportional. The comparison between the signals $\Pi(t)$ and $R_{H} Q(t)$ is presented in figure 7 over an oscillation period of one second.

Figure 7 shows the comparison for three different flows. The graphs on the left show the direct comparison between the signals $\Pi(t)$ and the product $R_{H} \cdot Q(t)$. The graphs on the right correspond to the same experiments; they assess the linearity between the filtration velocity and the pressure drop. In these graphs, the experimental data (blue dots) is compared to the straight line of equation $v(t)=\Pi(t) /\left(R_{H} S_{0}\right)$, which corresponds to the measurement.

The results shown in figure 7 (a) represent the majority of the experiments described in this article. The Reynolds number is 2.11 and the signal $R_{H} Q(t)$ matches the pressure signal $\Pi(t)$ accurately. The filtration velocity and the pressure drop are linearly related, as indicated by the value of the linear regression coefficient between the two signals $R^{2}=0.9994$. These observations indicate that the experiment is in a Darcy's flow regime and justifies the analysis presented in section 2 .

The results shown in figure 7 (b) and 7 (c) correspond to some flows at low and large Reynolds numbers (respectively $R e=0.40$ and $R e=21.2$ ). They correspond to the limits of the experimental method described in this paper. In both cases, the signal $R_{H} Q(t)$ slightly lags the pressure drop, and some deviations to the linearity between $v(t)$ and $\Pi(t)$ are observed. These deviations are however small (the linear regression coefficients are respectively $R^{2}=0.9914$ and $R^{2}=0.9948$ ), which explains that permeability measurements in these flow regimes still correspond to the measures performed by drainage. The loss of the linearity between $v(t)$ and $\Pi(t)$ proves to be due to a small phase shift $\varphi$ between these two signals. The results presented in table 4.1 indicate that the phase shift increases in absolute value when the flow rate and the pressure signals are less correlated. This is indeed observed in experiments performed at high and low Reynolds number which indicates the limits of validity of this measuring technique.

These limits at low and high Reynolds number have different origins. It is indeed expected to observe deviations to Darcy's law at high Reynolds number as the flow becomes subject to inertia $[1,14]$. An other point is that these flows are generated through the porous media with the lowest permeabilities. Although the values of the cross-correlation coefficient $\gamma^{2}$ indicate that the quasi-static approximation still applies, some subtle transient effects start to appear. The phase shift between the velocity and the pressure signals increases indeed as the characteristic frequency $f_{\tau}$, defined in section 2.5 , gets closer to $f_{0}$. On the other hand, the flows at low Reynolds number correspond to experiments performed with the smallest 

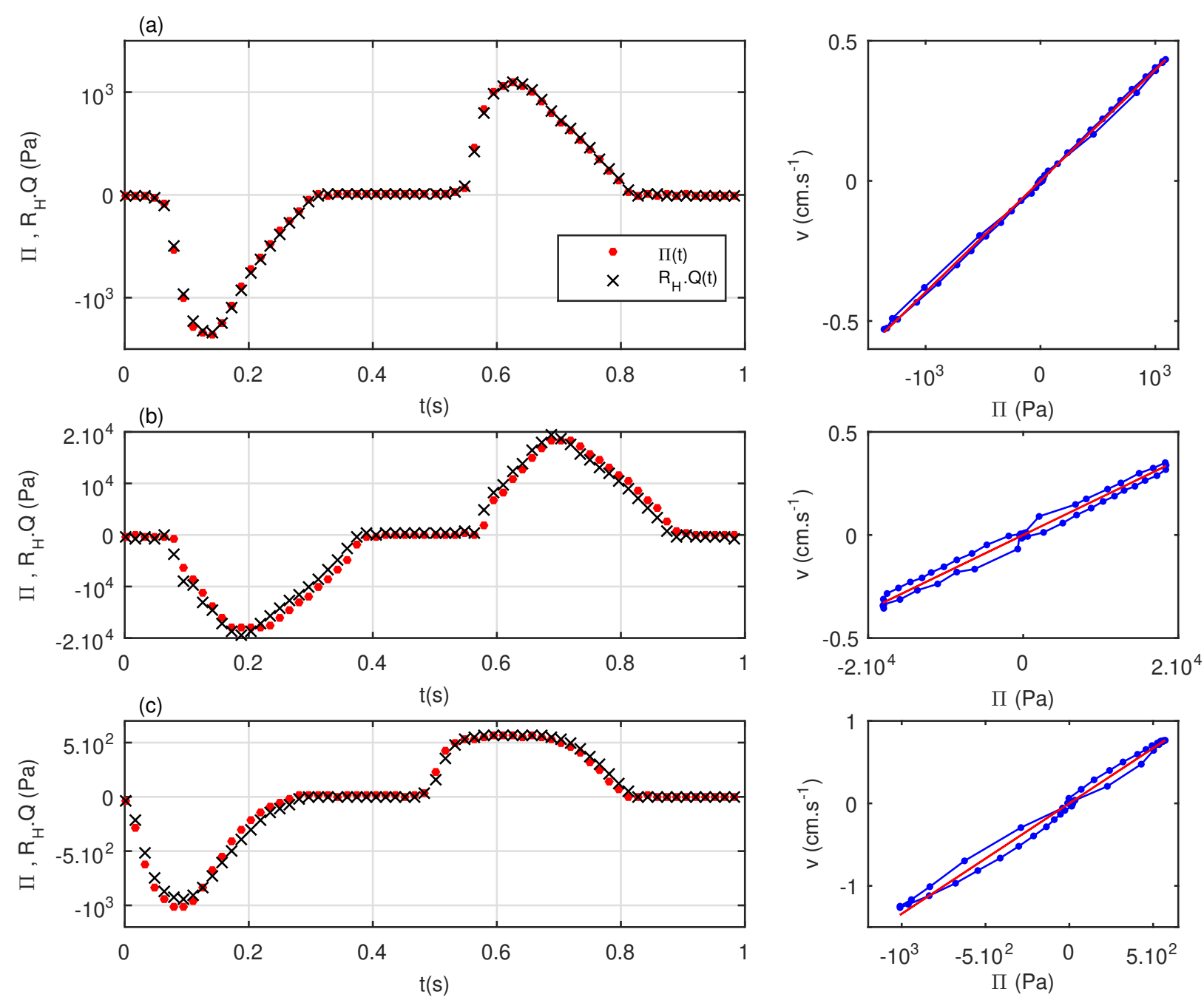

Fig. 7 Comparison between the pressure signals $\Pi(t)$ (red dots) and the product $R_{H} \cdot Q(t)$ computed with equations 13 and 14 (black crosses) over an oscillation period of one second. The graphs shown on the left compare the signals in time and the plots on the right assess the linearity between the filtration velocity and the pressure drop of the same experiment. In these graphs, the blue dots are the experimental data, compared to the red line of equation $v(t)=\Pi(t) /\left(R_{H} S_{0}\right)$ which corresponds to the measurement. a) Experiment performed with the glass beads $\mathrm{D}$ at $R e=3.1$. b) Experiment performed with the glass beads A at $R e=0.4$.

c) Experiment performed with the glass beads $\mathrm{G}$ at $R e=21.2$. 
beads, which generate the smallest permeabilities. In these cases, the pressure drop generated to move the fluid becomes so high that a few beads could be displaced at the edges of the compacting meshes. This means that the compaction of the beads was not optimal for these sizes, although a significant portion of the beads was well packed and clearly dominated the permeability measurement. Another artifact of the flows generated through the smallest beads was that the pressure drop was high enough for the compliance or elasticity of some components (tubes, joints, etc...) of the apparatus to introduce a small phase lag between the displacement of the piston and the response in flow velocity. However, the effect of these limiting phenomena at high and low Reynolds number on the measurements was quite limited, which allowed determining the permeability of the porous medium to leading order and with little impact of the flow regime, as can be seen in figure 8 .

Figure 8 collates all the signals measured for different bead sizes at several oscillation amplitudes. As observed in figure 7, the filtration velocity varies linearly with the pressure drop for most of the experiments. Deviations are observed at the highest and lowest values of the Reynolds number. The permeability measurements are not impacted by the flow regime as long as the Reynolds number remains smaller than 10. Indeed, for the packed beads A to $\mathrm{F}$, the measurements of $K_{0}$ present little variation and no dependency with the Reynolds number. This is not the case for the measurements performed with the biggest beads, as there is a slight decrease of the permeability values for $R e \geq 10$, which indicates a deviation to Darcy's law in this regime [1].

Finally, the comparison between the signals $\Pi(t)$ and $R_{H} Q(t)$ on one hand ${ }^{2}$, and the verification of the linearity between $\Pi(t)$ and $v(t)$ on the other hand confirm the validity of the experimental technique described here as long as the flows are in Darcy's regime that is for Reynolds numbers below 10.

\section{Conclusion}

In this article, we have presented a new experimental technique and an original analysis for measuring the permeability of a porous medium using oscillating flows. In particular, this technique differs from other methods using oscillating flows $[12,13]$ by the spectral analysis presented in section 2 and the amplitude of the displacement of the fluid in the porous medium. This kind of experiment has appeal for systems in which the liquid is at equilibrium (chemically or thermally) with the medium itself and for which is is important to limit the magnitude of the induced fluid motion so as to limit flow-driven reactions within the medium. The setup is versatile and produces accurate permeability measurements with a relative precision of the order of $3.7 \%$. The analysis of the measured signals allows an interesting charactarisation from Darcy's flows to the inertial regime. This experimental technique will be used in further studies with porous media in equilibrium with their liquid phase.

\footnotetext{
2 This could have also be done in the frequency domain, as shown in appendix B
} 


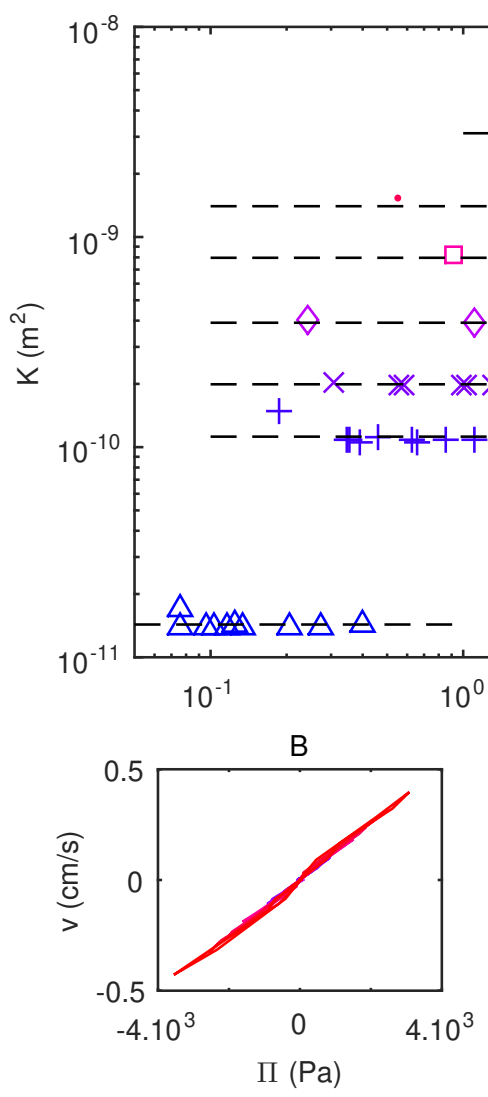

E
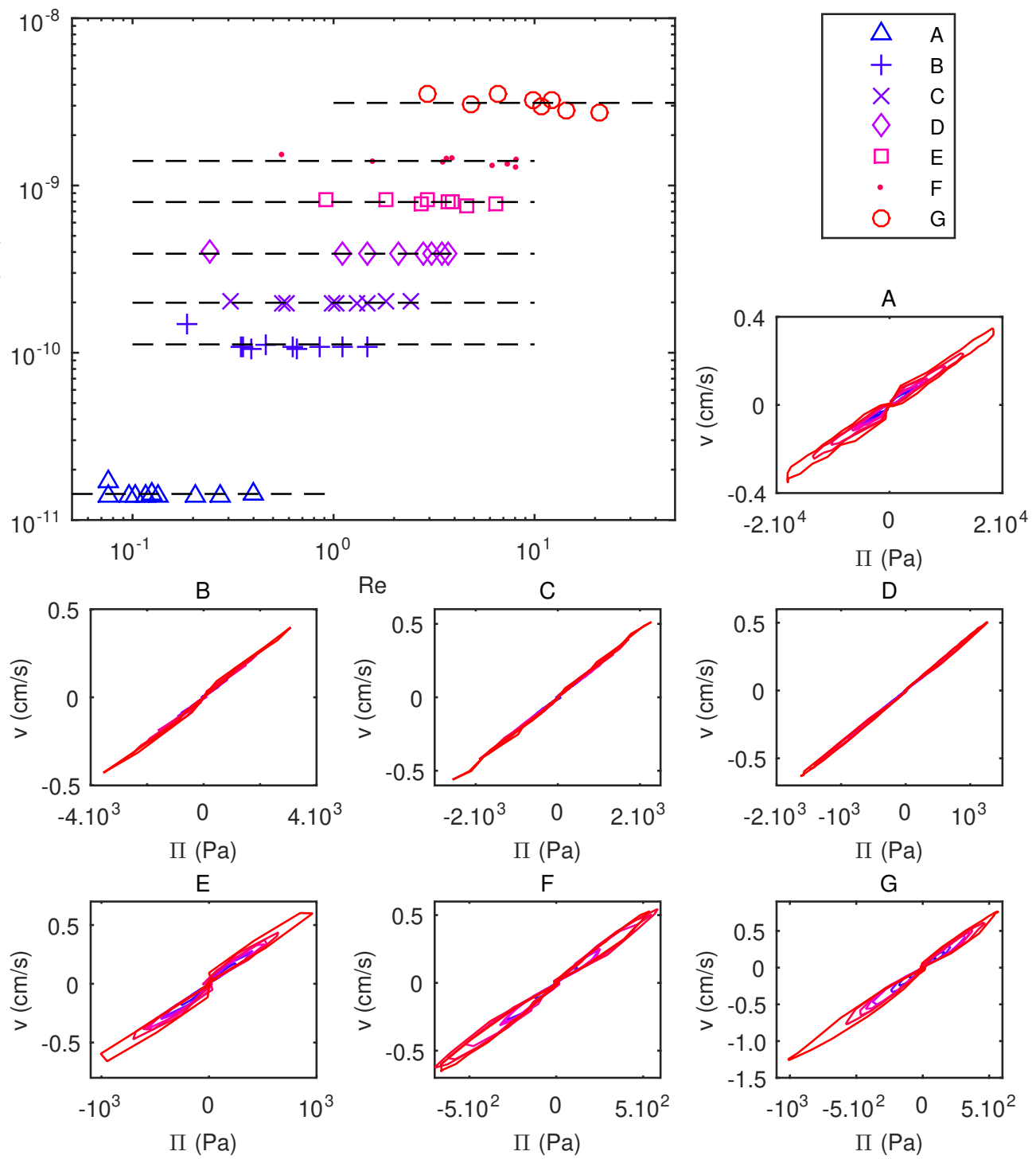

$\mathrm{Re}$

C
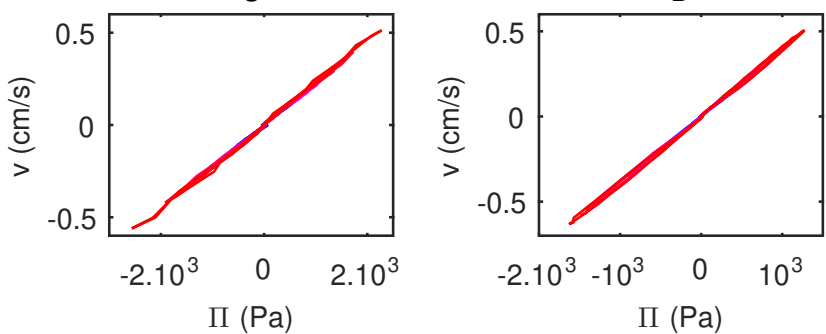

$\mathrm{F}$

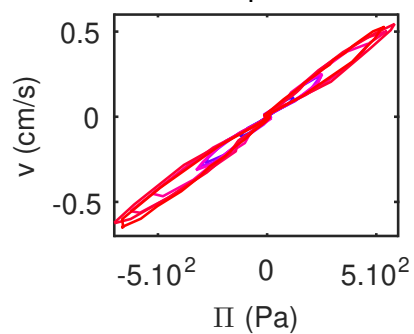

$\mathrm{G}$

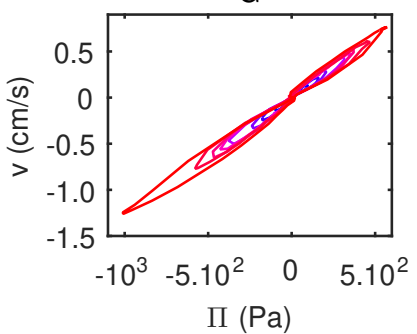

Fig. 8 Main plot: global results of all the permeability measurements for several packed beads (denoted from A to $\mathrm{G}$ as defined table 1). a) Permeability measurements for several Reynolds numbers. The measured permeabilities do not depend on the Reynolds number provided it is smaller than 10. For $R e \geq 10$, the measured values decrease with $R e$ as a consequence of inertial effects. Subplots: phase planes of the measurements for the different packed beads. The measurement of the filtration velocity $v$ depends linearly on the effective pressure drop with an excellent agreement for beads B, C, D and still with a good agreement $\left(R^{2} \geq 0.95\right)$ for the other glass beads. 


\section{aknowledgements}

JAN aknowledges funding from a Royal Society University Research Fellowship. This work was funded by grants from the British Antartic Survey Fundation and the Isaac Newton Trust. BG would like to warmly thank David Page-Croft and Paul Mitton for their expertise, help and friendly presence during this work.

\section{A Drainage experiments}

In this appendix, we describe complementary drainage experiments, performed using essentially the same setup as was used for the oscillatory experiments. These experiments were used to compare the results with a classical technique for which the flow is unidirectional and driven by gravity. Indeed, the versatility of the setup allows us to compare both measurements without changing the configuration of glass beads. The drainage experiments were performed after each oscillating run to compare both measurements accurately.

Experiments A reservoir is placed near the top of the flow cell and communicates with it through a siphon. This allow us to increase the volume of water flowing through the porous medium significantly and to perform reliable experiments. The diameter of the upper cylinder (cyl. B in figure 1) is actually smaller than that of the other cylinder to leave enough room for the siphon. The piston is fixed during the experiments and the only relevant recording with the acquisition card is the pressure signal. The drainage starts when the draining tap at the bottom of the flow cell is open (see figure 1). The flow is then unidirectional and the flux is measured with a weighing scale (Ohaus) placed at the outlet of the draining tap. The scales are connected to a computer which records the measurement with an acquisition frequency of $2.9 \mathrm{~Hz}$. The signals are then analyzed using the procedure described in the next paragraph.

Processing The pressure balance and the analysis principle remain the same as the ones described in section 3.1. However, since the flows are not periodic for these experiments, we do not compute $\Pi$ by subtracting the mean value of $\Delta P(t)$, but by subtracting the hydrostatic pressure difference recorded when there is no flow. Contrary to the oscillating measurements, the sampling rates of the signal $V(t)$ (measured with the scales) is much smaller than the sampling rate of the acquisition of $\Pi(t)$. We interpolate $V(t)$ with a cubic spline on the time intervals of $\Pi(t)$. Since differentiating $V(t)$ would increase the uncertainties of the measurements, we rather integrate $\Pi(t)$ numerically to fit the signal of $V(t)$. Indeed, integrating equation 10 leads to $\int_{0}^{t} \Pi\left(t^{\prime}\right) d t^{\prime}=R_{H} \cdot V(t)$ which allows us to measure $R_{H}$ with a single parameter fit.

The result of the processing is shown in figure 9. The fit is plotted in the top graph and we check afterwards the good match of the values of $\Pi(t) / R_{H}$ and $Q(t)$ by differentiating the raw data of $V(t)$ (bottom graph). Note that the fit is performed on the points recorded between $1.25 \mathrm{~s}$ after the opening and $1.25 \mathrm{~s}$ before the locking of the draining tap. In the example showed in figure 9, the standard error associated with $R_{H}$ was calculated using Matlab was five orders of magnitude below its value. The main source of uncertainty associated with this measurement actually comes from the data recorded by the scales, which leads to a relative precision of the order of $3 \%$ for these measurements.

\section{B Analysis of the signal Harmonics}

The verification of the linearity between $\Pi(t)$ and $Q(t)$ presented in section 4.2 could also be done in the frequency domain. Since both signals are periodic with a period $1 / f_{0}=1 s$, they can be easily decomposed in harmonics of frequencies $i . f_{0}$ and whose amplitudes are denoted $\pi_{i}$ and $q_{i}$ respectively by FFT. Equation 10 implies the relationship

$$
\pi_{i}=R_{H} q_{i}
$$




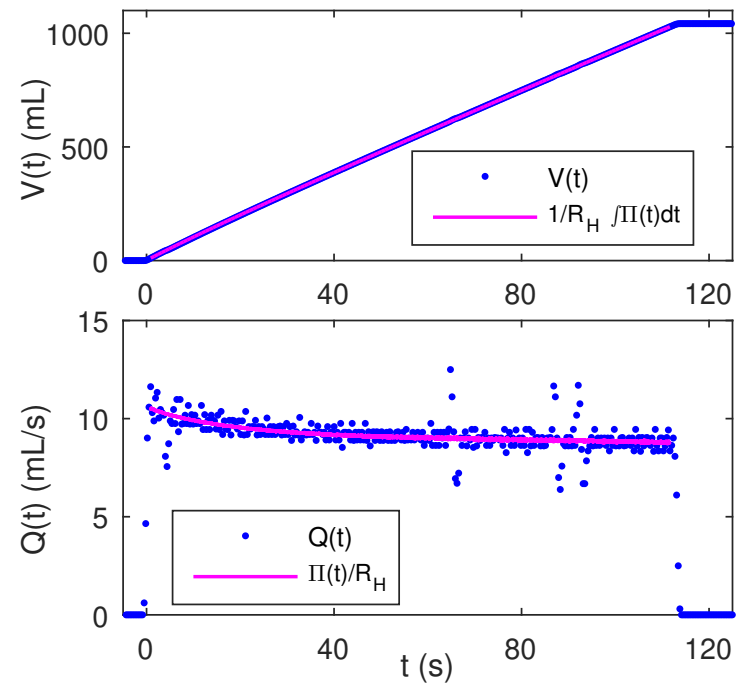

Fig. 9 Measurements of the hydraulic resistance from drainage experiments performed with $1 \mathrm{~mm}$ diameter glass beads (E). Top: fit of the integrated signal $\left(1 / R_{H}\right) \int_{0}^{t} \Pi$ (magenta line) to the measurements of $V(t)$ (blue dots) with $R_{H}$ as a fitting parameter. We find $R_{H}=$ 2.3.10 ${ }^{8}$ Pa.s. $m^{-3}$. Bottom: validation of the fit by comparing the values of $Q(t)$ computed with numerical differentiation of $V(t)$ (blue dots) with the scaled signal of $\Pi(t) / R_{H}$ (magenta line).

The spectral analysis shown in section 3.2.2 enables the identification of the harmonics of the pressure and flux signals and therefore a check on relationship 18. However, since the signals are subject to some noise of a peak to peak amplitude of $9 \mathrm{mV}$, we only considered here the harmonics whose amplitudes were greater or equal to $10 \mathrm{mV}$, and whose frequencies were smaller than $25 \mathrm{~Hz}$ as the harmonics are better defined in this frequency range (see figure 5). The results shown in figure 10 gather all the data collected per porous medium. The results indicate that the linearity between the harmonics $\pi_{i}$ and $q_{i}$ is indeed verified as a consequence of Darcy's law in the Fourier space.

\section{References}

1. Jacob Bear, Dynamics of fluids in porous media, Dover Publications Inc, 1972 New York

2. R. D. Wyckoff, H. G. Botset, M. Muskat, and D. W. Reed, The Measurement of the Permeability of Porous Media for Homogeneous Fluids, Review of Scientific Instruments 4, 394-405 (1933)

3. Thibault Candela, Emily E. Brodsky, Chris Marone, Derek Elsworth, Laboratory evidence for particle mobilization as a mechanism for permeability enhancement via dynamic stressing, Earth and Planetary Science Letters, 392, 279-291 (2014)

4. J. S. Wettlaufer, M. Grae Worster and Herbert E. Huppert, Natural convection during solidication of an alloy from above with application to the evolution of sea ice, Journal of Fluid Mechanics, 344, 291-316 (1997)

5. Johannes Freitag, Hajo Eicken, Meltwater circulation and permeability of Arctic summer sea ice derived from hydrological field experiments, Journal of Glaciology, 49, 349-358 (2003)

6. K. M. Golden, S. F. Ackley, V. I. Lytle, The Percolation Phase Transition in Sea Ice, Science, 282, 2238-2241 (1998) 


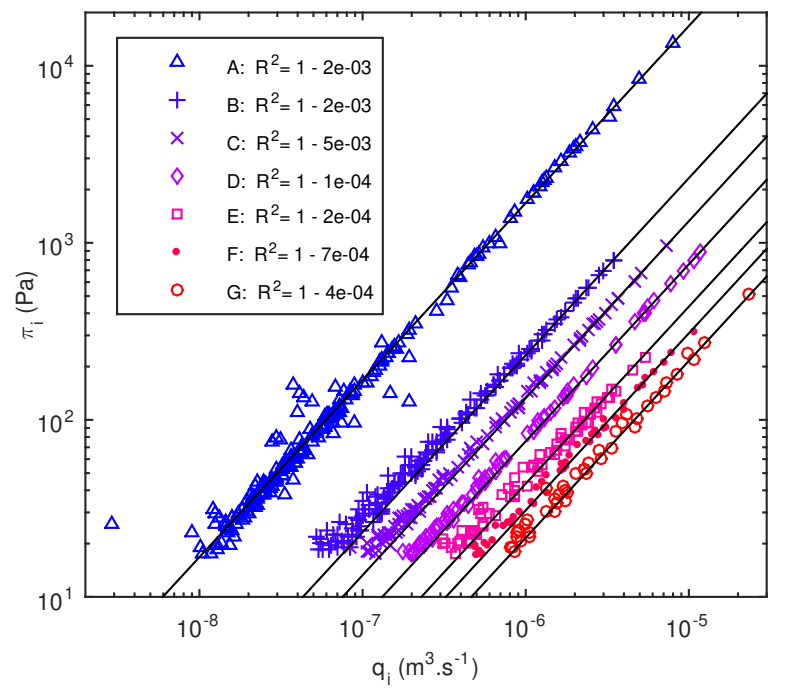

Fig. 10 Comparison of the amplitudes of the harmonics of frequencies $i . f_{0}$ of the pressure $\left(\pi_{i}\right)$ and flow rate $\left(q_{i}\right)$ signals. All the harmonics gather on the same straight line for each set of packed beads as a consequence of Darcy's law in the frequency space. The legend indicates the values of the linear regression coefficient computed for each porous medium.

7. K. M. Golden, H. Eicken, A. L. Heaton, J. Miner, D. J. Pringle and J. Zhu, Thermal evolution of permeability and microstructure in sea ice, Geophysical Research Letters, 34, L16501 (2007)

8. B. Andreotti, Y. Forterre, O. Pouliquen, Granular media Between Fluid and Solid, Cambridge University Press, Cambridge (2013)

9. Data Physics SignalForce V20 technical documentation

10. A. G. Crosby, An assessment of the accuracy of admittance and coherence estimates using synthetic data, Geophys. J. Int., 171, 25-54 (2007)

11. Julius S. Bendat, Allan G. Piersol, Random Data Analysis and Measurement Procedures, Wiley 4th edition (2010)

12. Mehmet Turgay Pamuk, Mustafa Özdemir, Friction factor, permeability and inertial coefficient of oscillating flow through porous media of packed balls, Experimental Thermal and Fluid Science, 38, 134-139 (2012)

13. P. Suri, M. Azeemuddin, M. Zaman, A.R. Kukreti, J.-C. Roegiers, Stress-dependent permeability measurement using the oscillating pulse technique, Journal of Petroleum Science and Engineering, 17, 247-264 (1997)

14. Stephen Whitaker, The Forchheimer Equation: A Theoretical Development, Transport in Porous Media 25: 27-61, (1996)

15. H.F. Burcharth, O.H. Andersen On the one-dimensional steady and unsteady porous flow equations Coastal Engineering 24, 233-257, (1995)

16. A.V. Kuznetsov, D.A. Nield, Forced Convection with Laminar Pulsating Flow in a Saturated Porous Channel or Tube Transport in Porous Media 65:505-523, (2006)

17. K.R. Rajagopal, On a hierarchy of approximate models for flows of incompressible fluids through porous solids Mathematical Models and Methods in Applied Sciences, 171, 215-252 (2007)

18. Tao Zhu, Christian Waluga, Barbara Wohlmuth, Michael Manhart, A Study of the Time Constant in Unsteady Porous Media Flow Using Direct Numerical Simulation Transport in Porous Media 104:161-179, (2014)

19. Kambiz Vafai, Handbook of Porous Media, Third edition, CRC Press (2015) 